\title{
Identification of material properties of FRC using coupled modeling
}

\author{
P. Procházka, A. Kohoutková \& J. Vodička \\ CTU Prague and Association of Civil Engineers, Prague, Czech Republic
}

\begin{abstract}
In this paper identification of material properties in the vicinity of reinforcement of FRC is based on coupled modeling. It consists of the mutual comparison of experimental and mathematical models with the aim of obtaining a more accurate estimate of stresses in experiments and more reliable input data in the mathematical treatment. As the measurements on site are very expensive, experiments simulating the system of the concrete-surrounding medium are prepared in scale models in stands (basins with a glazed front side and a length of 2-6 m), where physically equivalent materials substitute the real ones. Based on similarity rules, very good agreement with reality is attained. Typical applications are found in tunnel construction and reinforcement of slopes using recycled reinforced concretes (waste of bricks and used concrete serve as an aggregate in new built concretes). In order to identify the most exacting location in the concrete, coupled mechanical pullout tests are carried out together with chemical analysis conducted by Raman spectroscopy. It appears that the extent of ettringite on the interface fiber-surrounding matrix plays a very important role, and also other minerals occurring there can influence the interface situation, but less than the ettringite. In the numerical treatment a useful trick is applied, which stems from the idea of generalization of temperature effects eigenparameters. They describe the plastic behavior as well as the damage at the interfaces. Their applications in the paper will be the most important element of the creation of the coupled model.

Keywords: coupled modeling, fiber reinforced concrete, recycled aggregate, chemo-mechanical analysis, eigenparameters; application: slope reinforcement.
\end{abstract}

\section{Introduction}

Fibers play a very important role, particularly during the curing process of concrete, as they suppress local cracking and warping in the composite structure 
and avoid the possibility of corrosion of reinforcing steel rebars. On the other hand, polypropylene fibers can display other advantages. If aggregate from recycled material (shattered bricks or concretes) are used in the concrete mixture, they essentially increase the toughness of the material and can be applied to the elements that are in tension. It also appears that after mobilization of the fibers, even higher peak stresses can be safely attained. A very important fact follows from numerous experimental studies: since the steel fibers increase the protection of complete concrete against flaws, the fibers in recycled concrete increase the tensile bearing capacity of the material. The pullout problem was carried out for both materials, as a chemo-mechanical analysis explained certain reasons of the behavior in both types of materials: classical concrete reinforced by stiff fibers and concrete recycled with polypropylene fibers. Convergence analysis for determining cohesion and tensile strength is proposed and eventually a nano-mechanical measurement based on Raman microscopy is introduced.

The testing machine MTS Alliance RT/30 was used for carrying out the mechanical tests. From the combination (coupling) of experimental and theoretical methods the possibility of looking into the heart of the problem, the interfacial fiber-concrete mechanical and chemical properties, is enabled. The interfacial mechanical characteristics are involved in the angle of internal friction and cohesion. With these mechanical characterizations the chemical spectra and, consequently, the description of chemical elements and minerals, express the connection of nano- chemo- and mechanical properties.

\section{Experiments with classical concrete}

The testing machine MTS Alliance RT/30, see Fig. 1, is used for the pullout tests that are carried out for the purpose of this study. It is an electromechanical tool for compressive, tensile, and bending tests of materials. The maximum compressive and tensile force is $30 \mathrm{kN}$. The size of the possible samples is $150 \mathrm{x}$ $150 \times 250 \mathrm{~mm}$ (width $\times$ length $\times$ height). The velocity of loading was in our case $0.04 \mathrm{~mm} /$ minute.

The scheme of the container in which the fiber-concrete aggregate samples have been tested is depicted in Fig. 2. In the container the cement paste with one fiber symmetrically positioned in the aggregate is cured. Six samples have been tested. The results in time of curing are given in Fig. 3. The experiment was prepared with a high quality of preparation of cement paste and the positioning of the fiber was also extraordinarily accurate. The results of this study testify to this, as the variance is very small. Fig. 4 shows the appropriate graph obtained from statistical averaging of the previous results.

We would probably be interested in the reason why the steel reinforced concrete loses its bearing capacity during the curing process. The answer may follow from the chemical test descriptions that are presented in the next section. In the case the steel fibers, or other polymerized fibers used in a humid milieu, similar results can be expected. The peak stresses are attained not at the end of the curing and hardening process of concrete, but early in the beginning. Our interest is concentrated exactly on the time interval when the mixture loses its water contents and this is the moment of the highest admissible stress. 


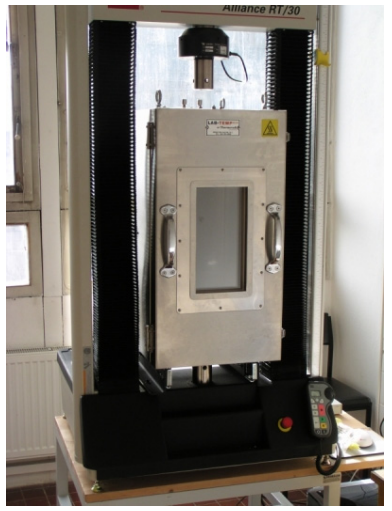

Figure 1: $\quad$ Testing machine.

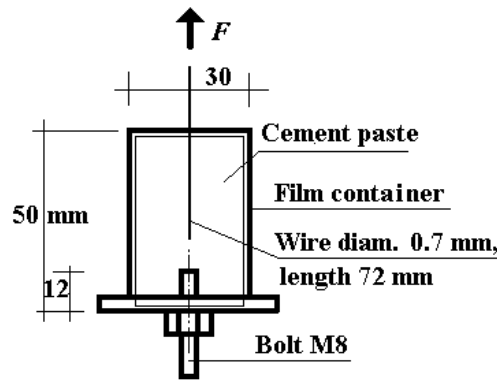

Figure 2: $\quad$ Scheme of the tested samples.

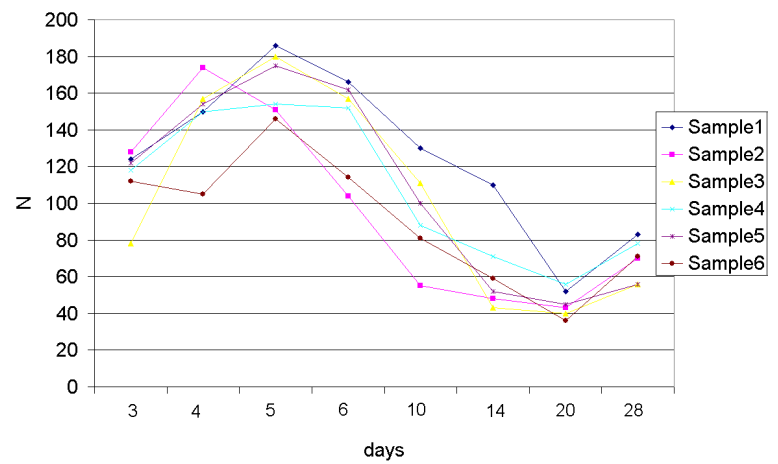

Figure 3: Admissible forces for six samples at the time of concrete curing.

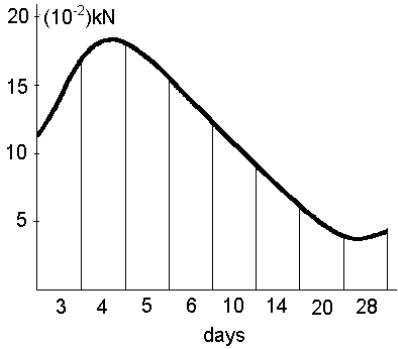

Figure 4: Resulting average admissible forces at the time of concrete curing. 


\section{Raman spectroscopy of cement-steel interfaces}

As mentioned above, it is widely accepted that the mechanical behavior of composites is highly dependent on the interface between the fiber and the matrix. The interface exists at some area around the fiber surface, where the local properties, including the morphological features, chemical compositions and thermo-mechanical properties, begin to change. The range of the microstructure and mechanical property gradients within the interface is from nanometers to micrometers. Several different test methods, such as the Raman spectroscopy stress field analysis, were used to investigate the interface properties. The microstructure of the paste matrix in the vicinity of the transition zone of the fibers is considerably different from that of the bulk paste away from the interface. It was observed that the transition zone in the mature composite is rich in $\mathrm{Ca}(\mathrm{OH})_{2}$, usually in direct contact with fiber surface, and is also quite porous, making it different from the microstructure of the bulk paste. The $\mathrm{Ca}(\mathrm{OH})_{2}$ layer is about $1 \mu \mathrm{m}$ thick and resembles the duplex films. Pullout tests were carried out for chemical and mechanical characterization of the bonding. It was found that frictional as well as anchoring effects controlled the pullout resistance of the straight fibers.

The application of Raman ad infrared spectroscopy in the field of cement and concrete chemistry are quite significant. Measuring the relative intensity of Raman peaks associated with $\mathrm{C}_{3} \mathrm{~S}$ and calcium hydroxide followed the progress of the reaction. These data sets show that a change in the hydration mechanism occurs at about 13 hours.

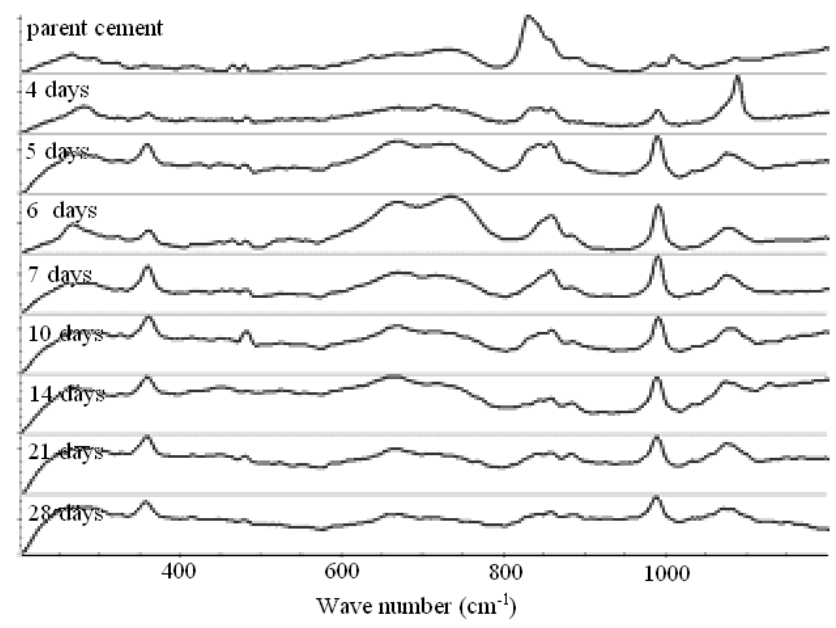

Figure 5: Raman spectra of the hydrated Portland cement during 0 to 28 days. 
Raman microspectroscopy chemical mapping has been used in this work, both for the monitoring of Portland cement hydration during 28 days and for spectroscopic analyses of differences in chemical composition of the interface area around the steel fiber surface in comparison with the surrounding bulk cement matrix composition (Fig. 5).

The five micrometer steps were selected for microspectroscopic mapping and the whole mapping area was $450 \times 450$ micrometers. The average spectrum has been computed from the set of measured mapped spectra. The hydration process is represented by a set of chemical equations describing the hydration of the main cement phases $\mathrm{C}_{3} \mathrm{~S}, \mathrm{C}_{2} \mathrm{~S}, \mathrm{C}_{3} \mathrm{~A}$ and $\mathrm{C}_{4} \mathrm{AF}$ (the nomenclature used here for cement is $\mathrm{C}=\mathrm{CaO}, \mathrm{S}=\mathrm{SiO}_{2}, \mathrm{H}=\mathrm{OH}, \mathrm{A}=\mathrm{Al}_{2} \mathrm{O}_{3}, \quad \mathrm{~F}=\mathrm{Fe}_{2} \mathrm{O}_{3}, \hat{\mathrm{S}}=\mathrm{SO}_{4}$ ). Raman microspectroscopy has been used as a powerful technique for the analysis of the hydration cement products, e.g. $\mathrm{Ca}(\mathrm{OH})_{2}$ and ettringite $\left(\mathrm{C}_{6} \mathrm{~A} \hat{\mathrm{S}}_{3} \mathrm{H}_{32}\right)$. Fig. 6 shows the Raman spectra of the hydrated Portland cement at 0 to 28 days period. The increasing intensity of $\mathrm{Ca}(\mathrm{OH})_{2}$ and ettringite and decreasing of the $\mathrm{C}_{3} \mathrm{~S}$ and $\mathrm{C}_{2} \mathrm{~S}$, respectively, is distinct. These chemical properties are probably the prevailingly reason for the decrease of the bearing capacity of the fibers imbedded in the concrete.

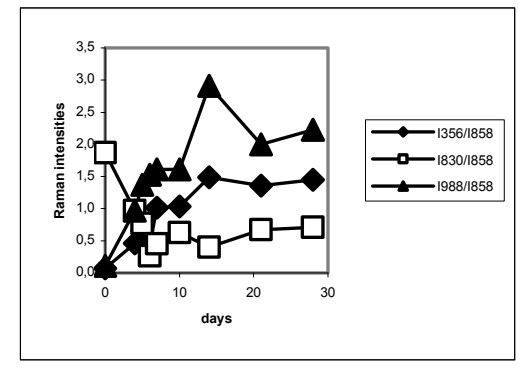

Figure 6: Changes in the relative abundance of $\mathrm{Ca}(\mathrm{OH})_{2}\left(\mathrm{I}_{356} / \mathrm{I}_{858}\right), \mathrm{C}_{3} \mathrm{~S}$ $\left(\mathrm{I}_{830} / \mathrm{I}_{858}\right)$ and ettringite $\left(\mathrm{I}_{988} / \mathrm{I}_{858}\right)$ versus days of the cement hydration.

\section{Recycled concrete and polypropylene fibers}

Among promising structural concretes one also can include ones that are created from recycling materials. They can substitute and spare the natural resources of gravel aggregates. Using additional scattered synthetic fibers, the structure of concrete is stiffened and former brittle material becomes ductile, which shows both high tensile strength and ductility. For concrete mixer creation, clean and unclean brick rubbish (waste) was used, i.e. structural rubbish in the second case contained imparted pieces of bricks, pore-concrete blocks, face bricks, floor tiles, ground concrete, backfill, and so on.

The strength of the materials strongly depends on the amount of cement. The following are certain material properties for various mixtures measured against the standard concrete M20: 
a) brick-concretes with lower amount of cement (denoted as C1T)

- tensile strength at bending

$5.24 \%$

- compressive strength

$10.24 \%$

- strength in transversal tension

$13.50 \%$

b) brick-concretes with higher amount of cement (denoted as $\mathrm{C} 2 \mathrm{H}$ )

- tensile strength at bending

$7.98 \%$

- compressive strength

$16.27 \%$

- strength in transversal tension

$19.47 \%$

Denotation $\mathrm{C} 3 \mathrm{~T}$ and $\mathrm{C} 4 \mathrm{H}$ refers to the same material properties after 90 days of use. Absolute values of the observed concretes with brick waste aggregates are illustrated in Fig. 7.

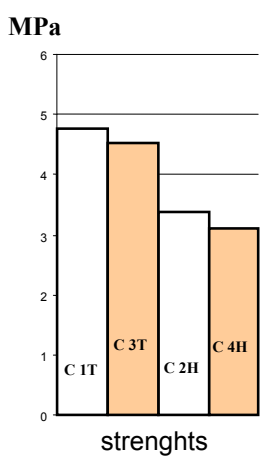

Tensile strength in bending

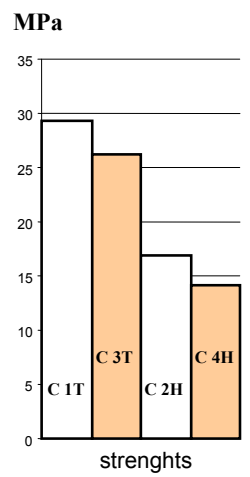

Compressive strength

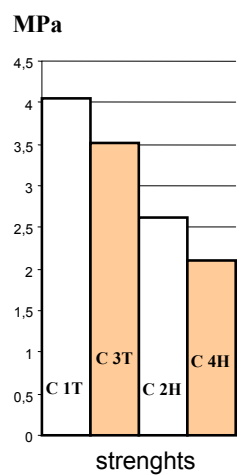

Lateral tensile strength

Figure 7: Comparison of basic characteristics of brick concretes with fibers.

The strength of brick concrete was lower than that of fine grain concrete, which is an impact of the inactive component, i.e. brick rubbish. Fibers scattered in the structure of brick concrete change the character of classical damage of trial bodies. Fibers also change the values of tensile strengths in bending under reloading by one or two concentrated loads. Last but not least, it is possible to produce the brick concrete without expensive admixtures. For tests on watertight concrete following the standard "Determination of water-tightness of concrete", samples from brick concrete with fibers $\mathrm{C} 3 \mathrm{~T}$ and $\mathrm{C} 4 \mathrm{H}$ from unclean brick rubbish aged 3 months were selected. Three cubes with sides of $150 \mathrm{~mm}$ were loaded in a watertight box for 24 hours by water pressure $0.1 \mathrm{MPa}$ and then another 24 hours by pressure $0.2 \mathrm{MPa}$. Leakage through the parallelepipeds attained nearly the upper surface of the cubes and the area of each shattered by 
lateral tension was more than $90 \%$ wet. With respect to the results of the tests, the water-tightness of the brick concrete with fibers is negligible.

For the typical structure of three types of concrete with waste brick aggregate, the pullout test results are seen in Fig. 8. Here the increase of admissible stress is obvious after the polypropylene fibers have been mobilized. A small deviation of force-displacement curves also indicates relatively reliable samples prepared for these tests.

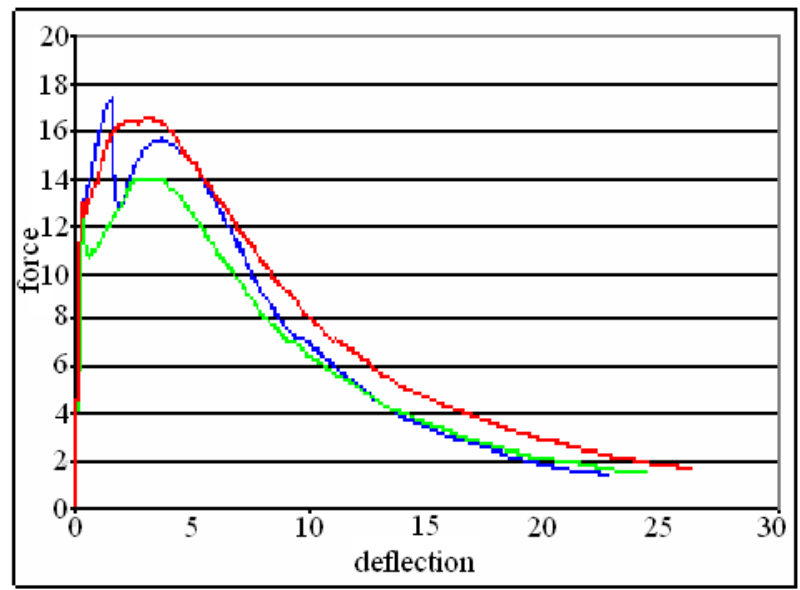

Figure 8: $\quad$ Diagram force $(\mathrm{kN})$ deflection $(\mathrm{mm})$ for recycled concrete.

\section{Mathematical formulation for the coupled modeling}

Because of the shape of the samples, the axisymmetric problem is solved. The displacements are described by a vector function $\boldsymbol{u} \equiv\left\{u_{r}, u_{\theta}\right\}$ of the variable $\boldsymbol{x} \equiv\{r, \theta\}$. The traction field on the interfaces or boundaries is denoted as $\boldsymbol{p} \equiv\left\{p_{r}, p_{\theta}\right\}$. Assuming the "small deformation" theory, it may be satisfactory to formulate the essential contact conditions on the interface as follows (no penetration conditions):

$$
[u]_{r}=u_{r}^{c}-u_{r}^{a} \leq 0 \quad \text { on } \Gamma_{C}
$$

where $\Gamma_{C}$ is the interfacial boundary between the fiber and the matrix, $u_{r}^{c}$ is the outward normal (radial) displacement of the fiber at a current point and $u_{r}^{a}$ is the same displacement at an adjacent point inside the concrete matrix on the interfacial boundary $\Gamma_{C}$. Similarly we define

$$
[u]_{t}=u_{t}^{c}-u_{t}^{a} \leq 0 \quad \text { on } \Gamma_{C}
$$


At each adjacent point on the interface $\bar{p}_{r} \leq p_{+}$has to be valid, where $p_{+}$is the tensile strength and $\bar{p}_{r}=p_{r}+\delta_{r}$, where $\delta_{r}$ is an internal parameter. Similarly introduce $\bar{p}_{t}=p_{t}+\delta_{t}$, where $\delta_{t}$ is another internal parameter. In this way, in the radial direction Fischera's conditions should be fulfilled:

$$
[u]_{r} \leq 0, \quad p_{+} \kappa\left(p_{+}-p_{r}\right)-p_{r} \geq 0, \quad\left\{p_{+} \kappa\left(p_{+}-p_{r}\right)-p_{r}\right\}[u]_{r}=0
$$

where $\kappa$ is the Heaviside function. In the tangential direction it should be valid that:

$$
\begin{gathered}
c \kappa\left(p_{+}-\bar{p}_{r}\right)-\bar{p}_{r} \tan \varphi-\left|\bar{p}_{t}\right| \geq 0, \quad\left|[u]_{t}\right| \geq 0, \\
\left\{c \kappa\left(p_{+}-\bar{p}_{r}\right)-\bar{p}_{r} \tan \varphi-\left|\bar{p}_{t}\right|\right\}\left|[u]_{t}\right|=0
\end{gathered}
$$

where $\tan \varphi$ is the tangent of the internal friction of both materials (Coulomb friction), $\tau_{b}$ is the shear strength or cohesion, both being given material coefficients that are different for different coupled materials on contact. These conditions describe the generalized Mohr-Coulomb law involving the exclusion of tension.

We concatenate the above conditions and generalize them to obtain a realistic model of the interfacial behavior. Then, the problem can be formulated in terms of penalties as coefficients of constraint (side conditions). Setting

$$
\bar{p}_{r}=k_{r}[u]_{r}, p_{r}=k_{r}[u]_{r}-\delta_{r} \text {, and } \bar{p}_{t}=k_{\theta}[u]_{\theta}, p_{t}=k_{t}[u]_{t}-\delta_{\theta},
$$

where $k_{r}, k_{\theta}$ are normal spring and tangential spring stiffnesses. The extended Lagrange principle provides ( $\Gamma$ is the external boundary and $a_{s}(\boldsymbol{u}, \boldsymbol{u})$ is the bilinear form of the system fiber concrete matrix):

$$
\begin{aligned}
\Pi=\frac{1}{2} & \sum_{s=1}^{2} a_{s}(\boldsymbol{u}, \boldsymbol{u})-\boldsymbol{\oint}_{\Gamma}^{\mathrm{T}} \boldsymbol{u} \mathrm{d} \boldsymbol{x}+ \\
& +\int_{\Gamma_{C}}\left\{k_{r}\left([u]_{r}\right)^{2}+k_{r}[u]_{r}\left|[u]_{t}\right|+k_{t}\left([u]_{t}\right)^{2}\right\} \mathrm{d} \boldsymbol{x}- \\
& -\int_{\Gamma_{C}}\left\{\left(p_{+}\right) \kappa\left(p_{+}-\bar{p}_{r}\right)[u]_{r}+c \kappa\left(p_{+}-\bar{p}_{r}\right)\left|[u]_{t}\right|\right\} \mathrm{d} \boldsymbol{x}
\end{aligned}
$$

Note that the spring stiffnesses $k_{r}, k_{t}$ play the role of penalty. The problem can also be formulated in terms of Lagrangian multipliers, which then leads to mixed formulation. The latter case is more suitable for a small number of boundary variables; the problem looked at here decreases the number of unknowns introducing the penalty parameters.

\section{Coupled modeling}

Considering the external boundary conditions and the material constants are given, the main objective here is to adopt the numerical results and the experimental conclusions. One possible approximation is the assumption that formulates the transformation formulas for interfacial forces. In the example 
presented hereinafter, the normal internal parameters $\delta_{r}$ are assumed too small in comparison to the real tractions and the tangential internal parameters are selected in such a way that $\delta_{t}=a p_{t}$, where the coefficient is to be determined from the condition: calculated external energy is equal to measured external energy.

As the assumption applied here is in fact very simple, the algorithm is easy. Calculate the response of the force applied in real situation and compare the results expressed in terms of external energy. Since most probably they will not be equal, calculate the internal parameter. This is not easy in this case as the relations between internal parameters and the energy are not linear. On the other hand, a smart algorithm can be used, such as the steepest descent or Raphson iteration. The material properties are selected as: $E_{\mathrm{f}}=170 \mathrm{GPa}, E_{\mathrm{m}}=17 \mathrm{GPa}$, $v_{\mathrm{f}}=0.3, v_{\mathrm{m}}=0.16$. The radius of the fiber is $0.6 \mathrm{~mm}$, the coefficient of Coulomb friction is 0.23 and the shear bond strength is $43.5 \mathrm{kPa}$.

Sample results are depicted in Fig. 9. It is seen that the normal tractions do not principally change, but the shear tractions are basically improved by the optimization. The optimal appears to be $a=1.76$.
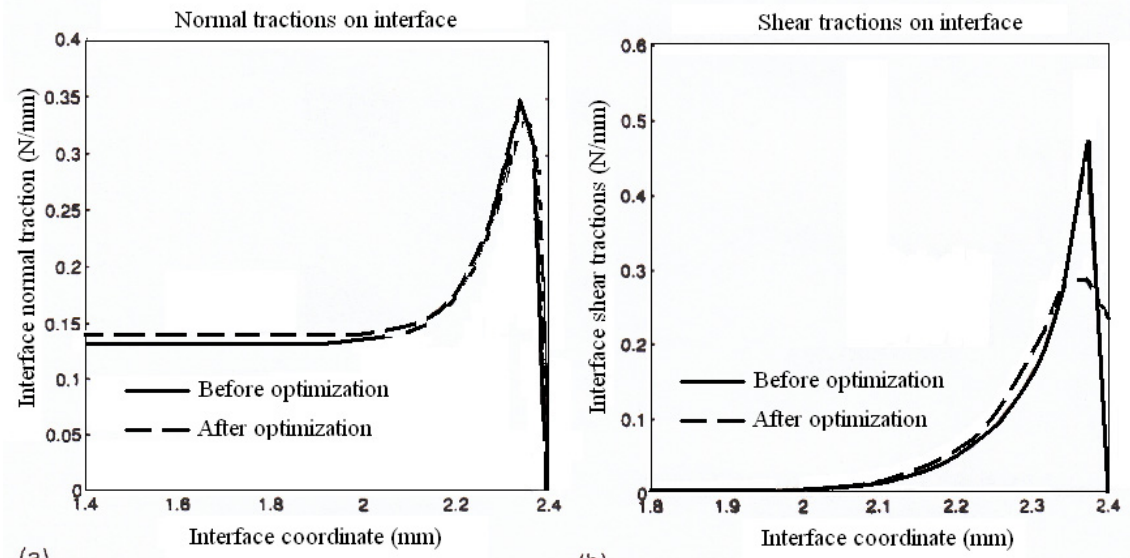

(b)

Figure 9: $\quad$ Interface tractions

\section{Possible application in increasing slope stability}

In this section the aim is focused on possible application of the recycled composite material in geomechanics. The reinforcement of slopes is very important in certain cases of extensive objects, deposits from open pit mines, tailing dams and such. If not stiffened, they occupy large areas of agricultural lots, or lots for dwelling buildings. In Fig. 10 the influence of nails from the material under consideration principally improves the slope stability. The distribution of vertical displacements for unreinforced (left picture) and reinforced (right picture) are depicted. Principal shear stresses with marked 
possible slip curves also illustrate the improvement of the stability situation using reinforcement by the recycled material (FRP). The final pictures are drawn in hypsography, which enables one to realize the stability situation before and after reinforcement. An unstable slope obviously turns to be stable when reinforcement is applied.
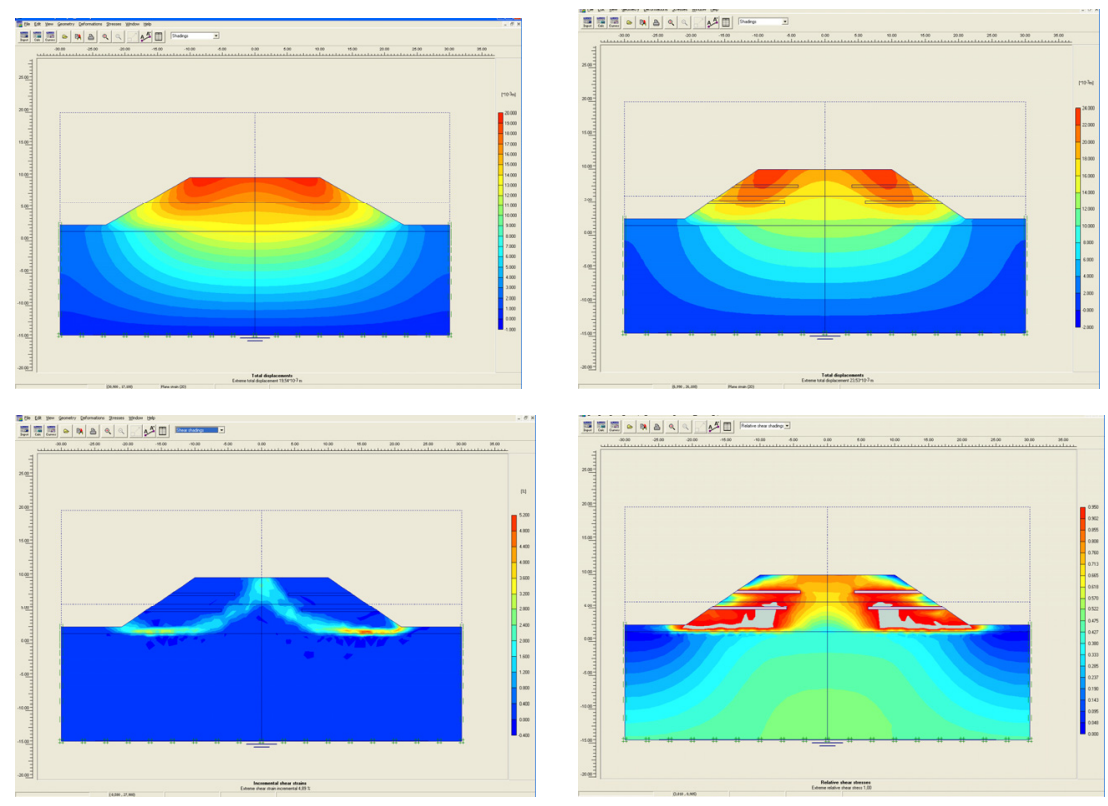

Figure 10: Displacements and principal shear stresses in unreinforced (left) and reinforced (right) slope.

\section{Conclusions}

In this paper chemical and mechanical properties are applied for investigation of contact zones in fiber reinforced concrete. The chemical treatment has been carried out by Raman microspectroscope, while the mechanical properties have been derived from results conducted in cylindrical samples (pullout test). It was shown that the results from both approaches should be observed simultaneously. Coupled modeling proved to be a powerful means for understanding the material behavior of the composite systems. Examples of practical applications of stiff and weak fibers have been presented.

\section{Acknowledgements}

The financial support of GAČR, project number 103/08/1197 is appreciated. The research has also been supported by a grant from the Ministry of Education of the Czech Republic number MSM6840770001,5. 